\title{
开放骨架磷酸铝合成反应数据库的建立与应用( I)
}

\author{
颜岩 ${ }^{(1)}$, 李激扬 ${ }^{(1)}$, 齐妙 ${ }^{(2)}$, 张晓 ${ }^{(1)}$, 于吉红 ${ }^{(1 *}$, 徐如人 ${ }^{(1) *}$ \\ (1) 无机合成与制备化学国家重点实验室, 吉林大学, 长春 130012; \\ (2) 东北师范大学化学学院, 长春 130117 \\ * 通讯作者, E-mail: jihong@jlu.edu.cn; rrxu@jlu.edu.cn
}

收稿日期: 2009-07-17; 接受日期: 2009-08-19

摘要本文介绍了开放骨架磷酸铝合成反应数据库的建立、收录的数据资料信息 和数据库的检索查询方法, 并对该数据库就骨架元素组成、反应产物的结构维数、 孔道环数、合成所用的有机模板剂等方面做了统计分析. 磷酸铝合成反应数据库的 建立对于微孔功能体系的分子工程学研究提供了重要的基础数据.

关键词

磷酸铝

开放骨架

合成

数据库

\section{1 引言}

自1982年美国U.C.C.公司成功开发出开放骨架 磷酸铝分子篮 $\mathrm{AlPO}_{4}-\mathrm{n}^{[1]}$ 以来, 开放骨架磷酸铝化合 物已成为多孔晶体材料的一个重要的家族. 磷酸铝分 子笁的骨架结构是由 $\mathrm{AlO}_{4}$ 四面体和 $\mathrm{PO}_{4}$ 四面体通过氧 桥严格交替连接构成, 其 $\mathrm{Al} / \mathrm{P}$ 比为 $1 / 1^{[2]}$, 其丰富的催 化、组装和吸附等性能引起人们极大的兴趣 ${ }^{[3,4]}$. 20 余 年来, 开放骨架磷酸铝的合成取得了巨大的进步, 目 前已有 60 余种磷酸铝分子篮结构被成功合成出来. 显著的例子是具有超大十八元环孔道的 $\mathrm{VFI}^{[5]}$ 和具有 手性十元环孔道的JRY ${ }^{[6]}$. 此外众多的具有阴离子骨 架的磷酸铝开放骨架化合物也被合成出来. 如具有 二十元环孔道结构的磷酸铝JDF-20 $0^{[7]}$ 和第一个具有 B-酸中心的AlPO-CJB1 $1^{[8]}$. 其结构可分为零维簇、一维 链、二维层和三维开放式骨架, 其 $\mathrm{Al} / \mathrm{P}$ 比可为 $1 / 1 、 1 / 2$ 、 2/3、3/4、4/5、5/6、6/7、11/12和12/13等 ${ }^{[9,10]}$.

无机微孔晶体材料的分子设计与定向合成是目 前国际微孔分子笁与分子工程学研究的重要前沿方 向. 然而, 由于无机微孔晶体化合物的合成化学十分 复杂, 至今人们对其生成机理还没有明确的认识, 这
就使这些材料的定向合成极具挑战性 $[11,12]$. 我们研究 组一直致力于特殊孔道结构多孔材料的分子工程学 研究, 并取得了一些研究成果. 例如, 开发设计了具 有特定孔道结构的分子篮骨架 ${ }^{[13 ~ 15]}$ 和具有特殊 Al/P比 磷酸铝骨架的计算机方法 ${ }^{[16]}$, 以及利用尺寸化学设 计超大孔开放骨架方法 ${ }^{[17]}$, 开发了以模板剂为导向 的定向合成路线等 ${ }^{[18 ~ 20]}$. 但是, 由于微孔晶体化合物 的晶化动力学受众多合成因素的影响, 其合成化学 非常复杂. 到目前为止, 人们对于其晶化机制还没 有清晰的认识, 因此, 利用传统路线进行这类化合物 的定向设计合成目前有很大困难. 深入研究水热合 成反应条件与产物结构之间的关系和规律对定向合 成具有特定结构的微孔化合物至关重要. 鉴于开放 骨架结构磷酸铝丰富的结构化学, 我们以其为研究 对象, 在大量合成研究的基础上, 在国际上率先建立 了磷酸铝合成反应数据库 ${ }^{[21]}$. 该数据库基于Linux平 台, 采用 $\mathrm{PHP}+\mathrm{Apache}+\mathrm{MySQL}$ 建立而成, 数据库包 含近 1600 条合成反应数据. 目前, 该数据库已被链接 到国际分子笁协会IZA(www.iza-structure.org/database $)^{[22]}$ 的网站上, 供全世界所有同行和学者免费使 
用. 我们希望借助各种计算机技术, 如数据挖掘技术

等, 开辟出微孔晶体材料定向合成的新路线.

本文将重点介绍磷酸铝合成反应数据库的建立 以及初步的查询及分析统计功能. 磷酸铝合成反应 数据库的建立有助于寻找开放骨架磷酸铝结构和合 成之间的关系. 为实现无机微孔材料和其他功能晶 体材料的定向设计合成开辟一条新的途径.

\section{2 实验部分}

以 Linux 作为服务器环境; Apache/2.2.8 (Linux) PHP/5.2.5 搭建 Web 服务器; PHP5.2.5 作为前台页面 编写语言; MySQL 4.1.7-Standard 作为后台数据库建 立磷酸铝合成反应数据库(Database of AlPO Syntheses). 编写工具使用 NotePad++直接调试代码对页面 以及整个构架进行整体设计和美化.

\section{3 结果与讨论}

\section{1 数据库构建及功能}

\subsection{1 系统框架}

磷酸铝合成反应数据库采用 MVC 模式进行整体 网站构架的设计, MVC 是 3 个单词的缩写：模型 (model)、视图(view)和控制(controller). MVC 模式可 以实现 Web 系统的职能分工, 具体体现为采用分层 结构进行设计. 网站整个系统的功能实现模块分为 数据访问层(包括实体和关联)、数据操作层(包括视图 和视图连接)、程序服务层(包括应用程序模块)、访问 使用层(包括网页汶览器窗口的表现形式和样式). 图 1 为网站构架模式示意图.

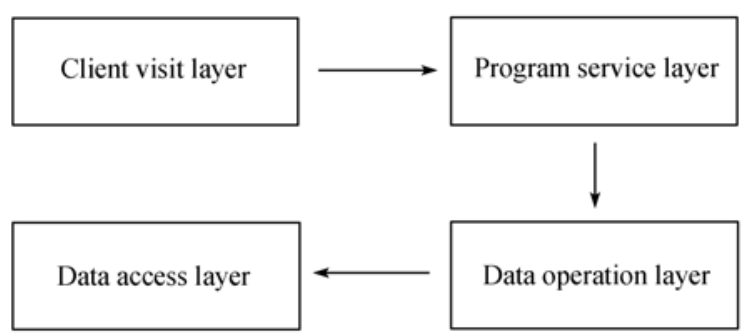

图 1 磷酸铝合成反应数据库构架模式示意图

\subsection{2 数据库结构设计}

磷酸铝合成反应数据库使用 MySQL 作为整个
网站的后台数据库, 依据数据挖掘所需要的基本数 据信息, 共设置 25 个可录入数据库的字段, 分成 5 个 不同的表，包括基本信息表 zeo_reference(记录文献 的出处等字段), 反应原料表 zeo_source_materials(记 录反应原料来源及纯度等字段), 原始配比表 zeo_batch_composition(记录反应凝胶组成等字段), 晶化条件表 zeo_crystallization(记录反应具体晶化条 件)和反应产物表 zeo_product(记录反应产物信息等 字段). 各表间通过主键和外键相互关联和约束.

\subsection{3 数据库界面设计}

磷酸铝合成反应数据库整体界面在设计时充分 考虑了查询、美观等方面因素. 保证网站前台显示界 面以及后台管理界面的整体风格一致性. 整体界面 设计使用 NotePad++作为代码编写工具, 直接调试 PHP 代码来达到界面的美化. 目前, 该数据库已连入 互联网并链接到权威机构国际分子篮协会 IZA 的网 站上(如图 2), 供全世界所有同行和学者免费使用.

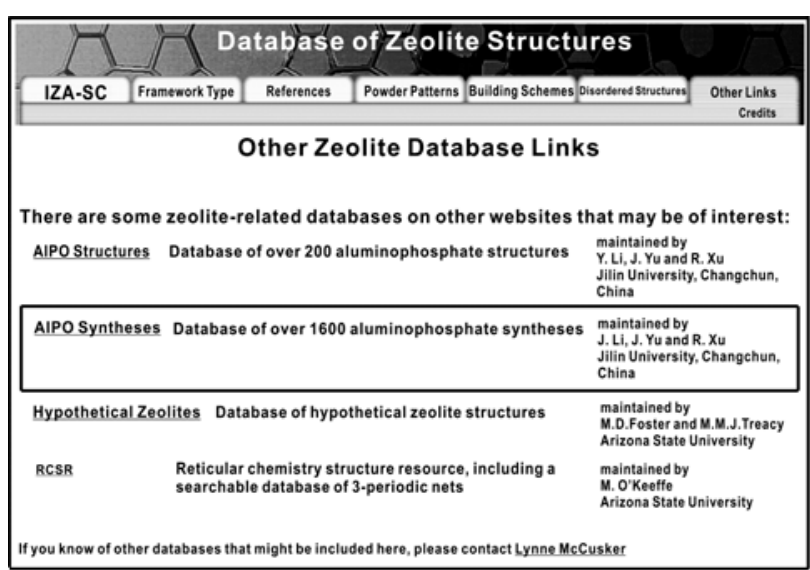

图 2 在 IZA 网站上的链接界面

数据库的整个界面(如图 3)分为 3 个部分. 包括 简介(introduction)、数据检索(data search)、检索帮助 (search help). 简介是对整个磷酸铝合成反应数据库 总体情况的简单介绍. 检索界面是整个网站前台页 面的核心部分. 查询帮助部分是对数据库检索的帮 助说明, 该部分详细阐述了不同字段检索时应遵循 的原则.

\subsection{4 数据库的检索功能}

磷酸铝合成反应数据库支持两种检索方式. 快 
速模糊检索(search)和高级检索(advanced search)(如 图 4). 高级检索分为 5 个检索区域: 文献检索区域, 包括对原始文献的标题、期刊、作者、卷、页码的检 索; 反应原料检索区域; 凝胶组成检索区域; 晶化条 件检索区域，包括晶化温度、晶化时间、 $\mathrm{pH}$ 值的检索;

\begin{tabular}{|c|c|c|}
\hline & \multicolumn{2}{|c|}{$\begin{array}{l}\text { Database of AIPO Syntheses } \\
\text { Prof. Jiyang Li, Prof. Jihong Yu, Prof. Ruren Xu } \\
\text { Molecular Engineering Group, Jilin University, P.R. China } \\
\text { Email: jihong@jlu.edu.cn(J. Yu) rrxu@jlu.edu.cn(R. Xu) }\end{array}$} \\
\hline Introduction & Data Search & Search Help \\
\hline \multicolumn{3}{|c|}{$\begin{array}{l}\text { Since the discovery of aluminophosphate molecular sieves, } \\
\text { designated AIPO4-n, in the early } 1980 \text { s by Wilson et al. at Un } \\
\text {-ion Carbide, there has been much progress in the synthesis } \\
\text { of open-framework aluminophosphates. A large number of } \\
\text { frameworks, ranging from neutral to anionic, have been prep } \\
\text {-ared.The AIPOs family, with its rich variety of structural } \\
\text { architectures, is an important one in the area of zeolites and } \\
\text { related open-framework inorganic materials. These materials } \\
\text { are typically prepared under hydrothermal or solvothermal } \\
\text { conditions,and many variables affect which framework type } \\
\text { is formed.These include the source materials, the batch } \\
\text { composition, the pH value, the template, the solvent and the } \\
\text { crystallization temperature and time. In this synthesis datab } \\
\text {-ase, we have collected more than } 1600 \text { reaction data from the } \\
\text { literature for over } 200 \text { AIPO structures. Each entry in the } \\
\text { database includes the literature reference, and detailed info } \\
\text {-rmation about the source materials, the template, the synth } \\
\text {-esis conditions, and the structural characteristics.This syn } \\
\text {-thesis database complements the AIPO structure database } \\
\text { ( http://www.iza-structure.org/databases, See Other Links ) } \\
\text { that is also maintained by our group. }\end{array}$} \\
\hline $\begin{array}{l}\text { Maintained by } \\
\text { Jilin Universit } \\
\text { Powered by ZE }\end{array}$ & Yan Tel: +86- & $\begin{array}{l}\text { 35168608, Fax: +86-431-85168608 } \\
\text { Changehun } 130012 \text {, P. R. China }\end{array}$ \\
\hline
\end{tabular}

图 3 磷酸铝合成反应数据库网站主界面

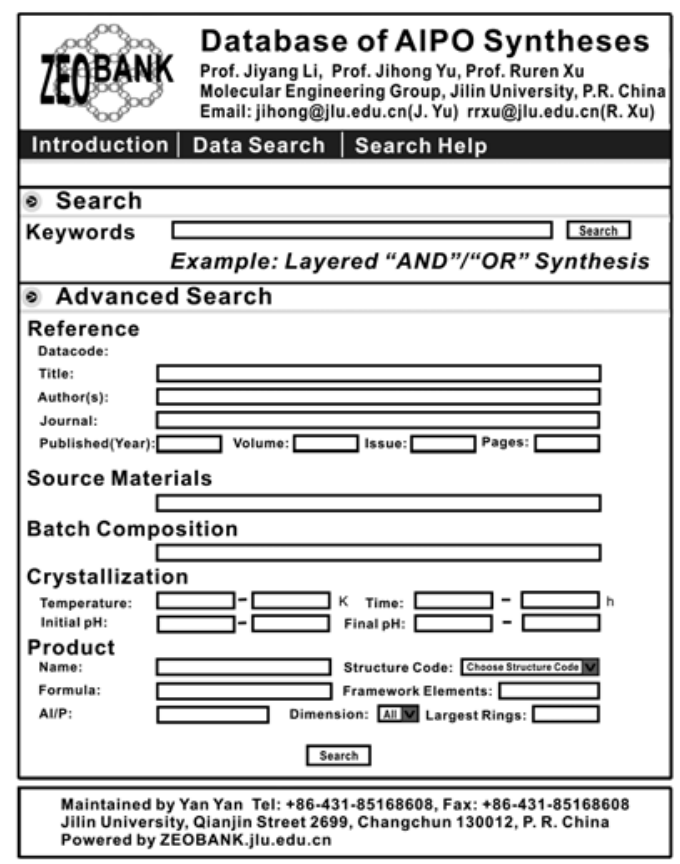

图 4 磷酸铝合成反应数据库检索部分主界面
反应产物检索, 包括化学名称、分子式、结构代码、 骨架元素、铝磷比、产物维数和最大孔道环数等字段 的检索. 磷酸铝合成反应数据支持多区域、多字段、 多关键字同时检索.

磷酸铝合成反应数据库有较强的数据提取功能. 符合检索条件的数据信息，分条显示在检索结果界 面中，并具体列出了文献作者、题目、文献出处、 文献ID号，符合检索条件的总数据条数、总页数等 信息.

此外，为方便使用，可以对查询结果以文献ID号 或发表时间等方式进行排序显示. 点击某条数据的 ID号或题目可查看该文献的详细信息(如图5).

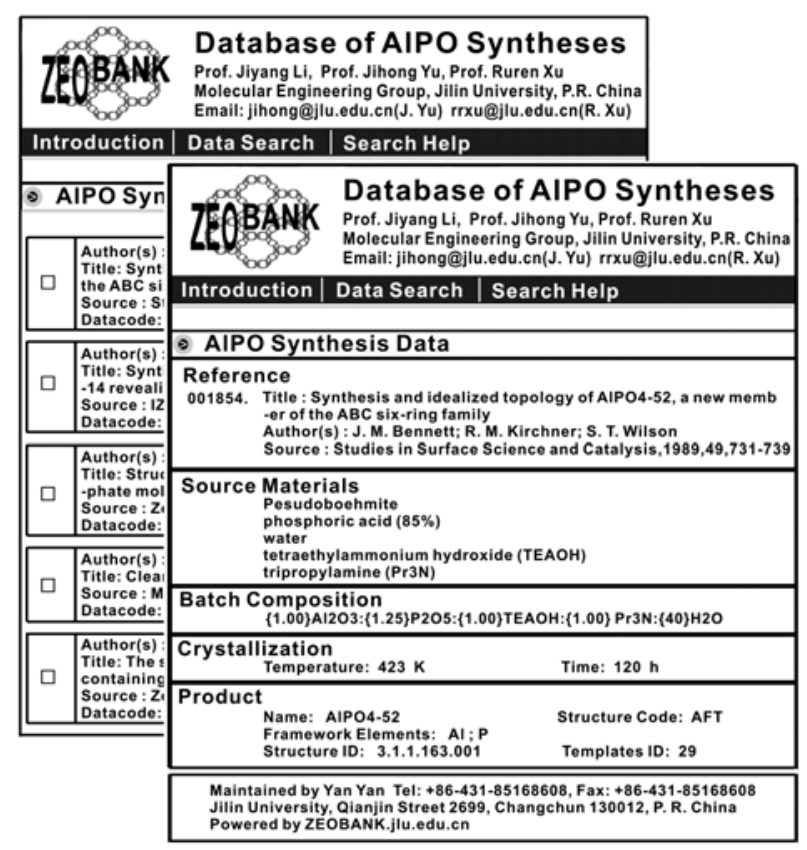

图 5 磷酸铝合成反应数据库检索 $\mathrm{AIPO}_{4}-5$ 的结果

\section{2 数据信息及统计分析}

磷酸铝合成反应数据库收录了 1936 年至今的大 量文献期刊、会议、学位论文、专利以及本实验室的 研究结果中提取出的 1585 条合成反应数据, 对应 230 余种磷酸铝骨架结构. 磷酸铝合成反应数据库的数 据在不断地更新, 它将为广大使用者提供最新最全 的磷酸铝合成数据资源.

我们对数据库数据做了如下几个方面的统计 分析: 


\subsection{1 孔道环数}

孔道大小是描述磷酸铝微孔结构的重要因素. 在磷酸铝合成反应数据库中, 按孔道窗口大小, 通常 将含有十四元环及以上的孔道称为超大孔, 含有十 二元环称为大孔, 含有十元环为中孔, 含有八元环及 以下为小孔. 图 6 给出了磷酸铝合成反应数据库中数 据按孔道环数分类的情况, 如图所示: 超大孔磷酸铝 合成数据有 63 条, 占总数据的 $4.60 \%$; 大孔磷酸铝合 成反应数据有 448 条占总数据的 $27.98 \%$; 中孔磷酸 铝合成反应数据有 151 条, 占总数据的 $9.43 \%$; 小孔 磷酸铝合成反应数据有 567 条, 占总数据的 $35.80 \%$; 其它的低维磷酸铝结构占 $22.11 \%$.

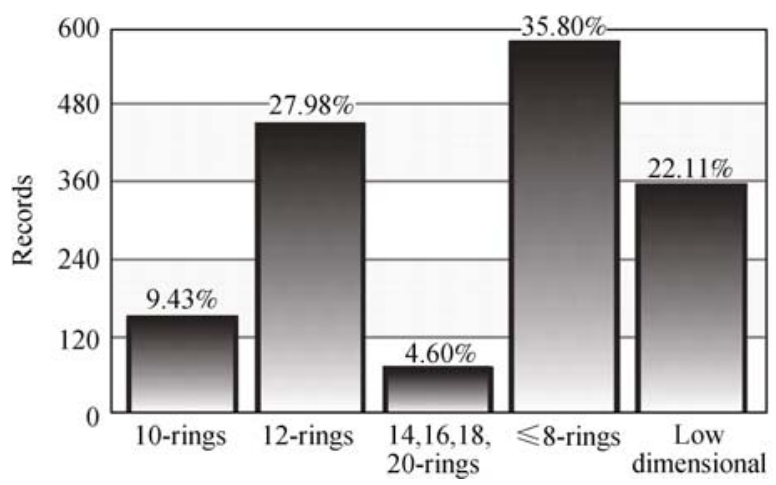

图 6 磷酸铝合成反应数据库中数据按孔道环数分类的分 布图

\subsection{2 骨架元素组成}

开放骨架磷酸铝是由 $\mathrm{AlO}_{n}(n=4,5,6)$ 和 $\mathrm{PO}_{4}$ 严格交 替形成的, $\mathrm{Al}$ 或P原子可以部分被 $\mathrm{Si}$ 或其他元素所取 代, 形成具有特殊性质的杂原子磷酸铝分子篮. 因此 磷酸铝合成反应数据库中还收录有含杂原子的磷酸 铝化合物, 骨架元素组成如图 7 所示, 含有纯 $\mathrm{Al}$ 和 $\mathrm{P}$ 元素的数据有 823 条, 占总数据的 $51.92 \%$; 其他元素 则以 $\mathrm{Si} 、 \mathrm{Co} 、 \mathrm{~V} 、 \mathrm{Mn} 、 \mathrm{Mg}$ 等常见. 其中骨架元素为 $\mathrm{Al} 、 \mathrm{P} 、 \mathrm{Si}$ 的数据有 242 条, 占总数据的分布比例为 $15.26 \%$; 骨架元素为 Al、P、Co的数据有 150 条, 占 总数据的分布比例为 $9.46 \%$; 而骨架元素为 $\mathrm{Al} 、 \mathrm{P}$ 、 $\mathrm{Mn}$, 或 $\mathrm{Al} 、 \mathrm{P} 、 \mathrm{Mg}$, 或 $\mathrm{Al} 、 \mathrm{P} 、 \mathrm{~V}$ 的数据分别有 47 、 $67 、 33$ 条, 分别占数据总分布比例的 $2.98 \% 、 4.23 \%$ 和 $2.82 \%$; 其余数据为 $\mathrm{Al} 、 \mathrm{P} 、 \mathrm{Xx}(\mathrm{Xx}$ 为不同于 $\mathrm{Si} 、 \mathrm{Co}$ 、 $\mathrm{V} 、 \mathrm{Mn} 、 \mathrm{Mg}$ 的元素), 共有数据 223 条, 占

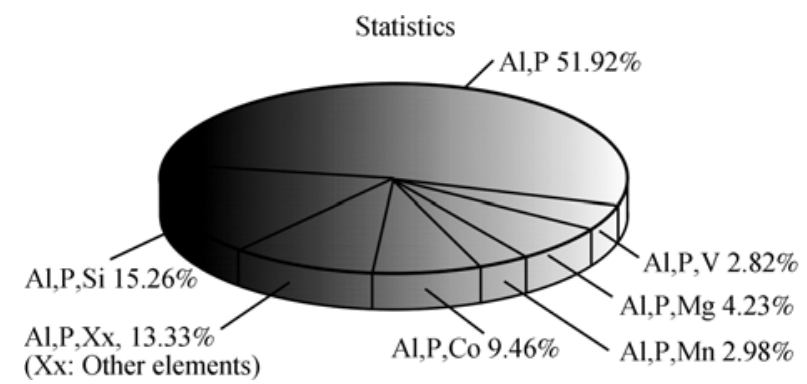

图 7 磷酸铝合成反应数据库中各种骨架元素的分布比例 总数据的 $13.33 \%$.

\subsection{3 反应产物的结构维数}

磷酸铝合成反应数据库中反应产物的结构按维 数可分为 $0-\mathrm{D}$ 簇, 1-D 链, 2-D 层, 3-D 开放骨架. 目前 数据库中收录的 1585 条数据中, $71.57 \%$ 的数据为 $3-\mathrm{D}$ 开放骨架结构, $12.32 \%$ 的数据为 2-D 层结构, 1-D 链数 据占 $3.98 \%, 0-\mathrm{D}$ 簇占 $12.13 \%$. 图 8 显示的是磷酸铝 合成反应数据库中产物维数的分布比例情况.

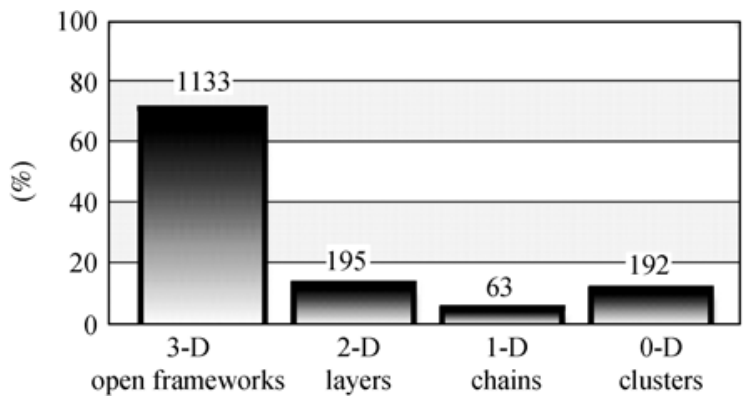

图 8 磷酸铝合成反应数据库中产物维数的分布比例

\subsection{4 模板剂}

在无机微孔晶体材料合成研究中, 模板剂的使 用与选择是至关重要的. 同样的原料配比及合成条 件, 采用不同模板剂常常得到不同结构的产物. 因此 从磷酸铝合成反应数据库中挖掘有关模板剂的信息 将是十分重要的工作.

统计结果显示: 在磷酸铝合成反应数据库中收录 了百余种有机模板剂, 包括单胺(mono-amine)、双胺 (di-amine)、多胺(poly-amine)和环状胺(cyclo-amine)等. 在单胺中以甲胺(methylamine)作为模板剂的有 48 条 记录. 以双胺中的乙二胺(ethylenediamine)为模板剂 
表 1 以二正丙胺 $\left(\mathrm{Pr}_{2} \mathrm{NH}\right)$ 为模板剂检索数据情况统计

\begin{tabular}{|c|c|c|c|c|}
\hline 序号 & 凝胶条件 & 反应产物 & 结构代码 & 数据(条) \\
\hline 1 & $\{1.80-2.20\} \mathrm{Pr}_{2} \mathrm{NH}:\{0.30\} \mathrm{MgO}:\{0.85\} \mathrm{Al}_{2} \mathrm{O}_{3}:\{1.0\} \mathrm{P}_{2} \mathrm{O}_{5}:\{50\} \mathrm{H}_{2} \mathrm{O}$ & MAPO-46(A-F) & AFS & 6 \\
\hline 2 & $\mathrm{Al}_{2} \mathrm{O}_{3}: \mathrm{P}_{2} \mathrm{O}_{5}: \mathrm{TiO}_{2}: \mathrm{i}-\mathrm{Pr}_{2} \mathrm{NH}: \mathrm{H}_{2} \mathrm{O}_{2}: \mathrm{C}_{6} \mathrm{H}_{14} \mathrm{O}_{4}$ & TiAPO-41 & AFO & 1 \\
\hline 3 & $\{0.5\} \mathrm{Al}_{2} \mathrm{O}_{3}:\{1.0\} \mathrm{P}_{2} \mathrm{O}_{5}:\{2.0\} \mathrm{Pr}_{2} \mathrm{NH}:\{48\} \mathrm{H}_{2} \mathrm{O}$ & AlPO-41 & AFO & 1 \\
\hline 4 & $\{1.0\} \mathrm{Al}_{2} \mathrm{O}_{3}:\{1.0\} \mathrm{P}_{2} \mathrm{O}_{5}:\{1.0\} \mathrm{Pr}_{2} \mathrm{NH}:\{80\} \mathrm{H}_{2} \mathrm{O}$ & VPI-5 & VFI & 1 \\
\hline 5 & $\{1.0\} \mathrm{Al}_{2} \mathrm{O}_{3}:\{1.0\} \mathrm{P}_{2} \mathrm{O}_{5}:\{1.0\} \mathrm{Pr}_{2} \mathrm{NH}:\{70\} \mathrm{H}_{2} \mathrm{O}$ & $\mathrm{AlPO}_{4}-11$ & AEL & 1 \\
\hline \multirow[t]{2}{*}{6} & $\{1.0\} \mathrm{Al}_{2} \mathrm{O}_{3}:\{1.0\} \mathrm{P}_{2} \mathrm{O}_{5}:\{0.6\} \mathrm{SiO}_{2}:\{1.0\}(\mathrm{TEA})_{2} \mathrm{O}:\{0.5\} \mathrm{Pr}_{2} \mathrm{NH}:\{50\} \mathrm{H}_{2} \mathrm{O}$ & SAPO-34 & CHA & 1 \\
\hline & $\{1.0\} \mathrm{Al}_{2} \mathrm{O}_{3}:\{1.2\} \mathrm{P}_{2} \mathrm{O}_{5}:\{0.3\} \mathrm{SiO}_{2}:\{0.5\}(\mathrm{TEA})_{2} \mathrm{O}:\{1.6\} \mathrm{Pr}_{2} \mathrm{NH}:\{52\} \mathrm{H}_{2} \mathrm{O}$ & SAPO-34 & CHA & 1 \\
\hline \multirow[t]{2}{*}{7} & $\{1\} \mathrm{Al}_{2} \mathrm{O}_{3}:\{1\} \mathrm{P}_{2} \mathrm{O}_{5}:\{0.3\} \mathrm{SiO}_{2}:\{1\} \mathrm{Pr}_{2} \mathrm{NH}:\{50\} \mathrm{H}_{2} \mathrm{O}$ & SAPO-31 & ATO & 1 \\
\hline & $\{1\} \mathrm{Al}_{2} \mathrm{O}_{3}:\{1\} \mathrm{P}_{2} \mathrm{O}_{5}:\{0.3\} \mathrm{SiO}_{2}:\{1\} \mathrm{Pr}_{2} \mathrm{NH}:\{30\} \mathrm{H}_{2} \mathrm{O}$ & SAPO-31 & ATO & 1 \\
\hline 8 & $\{1\} \mathrm{Al}_{2} \mathrm{O}_{3}:\{1.0-1.3\} \mathrm{P}_{2} \mathrm{O}_{5}:\{0.1-0.6\} \mathrm{SiO}_{2}:\{2.0-4.0\} \mathrm{Pr}_{2} \mathrm{NH}:\{60\} \mathrm{H}_{2} \mathrm{O}$ & SAPO-46 & AFS & 1 \\
\hline 9 & $\{0.9\} \mathrm{Al}_{2} \mathrm{O}_{3}:\{0.85\} \mathrm{P}_{2} \mathrm{O}_{5}:\{0.3\} \mathrm{SiO}_{2}:\{0.2\} \mathrm{CoO}:\{2\} \mathrm{Pr}_{2} \mathrm{NH}:\{55\} \mathrm{H}_{2} \mathrm{O}$ & CoSAPO-46 & AFS & 1 \\
\hline
\end{tabular}

的有数据 97 条之多. 多胺中二乙烯三胺(diethylenetriamine)和三乙烯四胺(triethylenetetramine)最为常用, 其相关的合成反应数据分别有 56 条和 42 条.

有机模板剂与无机开放骨架存在 3 种对应关系: (1)一种模板剂/多种结构; (2)多种模板剂/一种结构; (3) 一种模板剂/一种结构. 通过检索数据库中有机胺的 情况发现, 在开放骨架磷酸铝中, 一种模板剂/多种 结构与多种模板剂/一种结构的现象普遍存在. 例如, 以二正丙胺 $\left(\mathrm{Pr}_{2} \mathrm{NH}\right)$ 为模板剂, 可以合成出 9 种不同 的磷酸铝化合物，如表 1 所示.

另一方面，一些结构可由多种不同的模板剂得 到，它们对模板剂的依赖性很小. 以反应物 $\mathrm{AlPO}_{4}-5$ 为例, 检索得到的模板剂: 三丙胺、三乙胺、乙烯三 胺、四甲基氢氧化铵、 $N, N$-二甲基乙醇胺、四丙基氢 氧化铵、二环己胺、环己胺、2-甲基吡啶、六氢吡啶、 三乙醇胺、8-差基喹啉、 $N$-甲基环己胺、二乙醇胺、 三丁胺、二乙胺、1,4-二氮杂二环[2.2.2]辛烷、六亚 甲基四胺和甲胺等, 均可以合成 $\mathrm{AlPO}_{4}-5$ 分子篮.

有一些磷酸铝结构展现出较高的模板剂选择性, 只能由一种模板剂导向生成. 例如 $\mathrm{AlPO}_{4}-20$ (SOD)
在数据库中有 4 条记录, 全部是由四甲基氢氧化铵得 到; 同样 JDF-20 在数据库中有 12 条记录, 全部是由 三乙胺制得.

一些磷酸铝分子篮结构的生成是不同模板剂共 同作用的结果. 例如, 数据库中有 21 条记录显示四 丙基氢氧化铵和四甲基氢氧化铵作为混合模板剂可 以导向生成 SAPO-37.

\section{4 结论}

本文基于 Linux 平台, 利用 PHP+Apache+ MySQL 构架建立了开放骨架磷酸铝合成反应数据库. 数据库包含有 1585 条磷酸铝合成反应数据, 该数据 库具有查询、分析、统计等功能. 磷酸铝合成反应数 据库的建立为分子管以及相关多孔材料的定向合成 提供了重要的数据信息. 我们将对合成反应数据进 行参数化, 提取出重要的合成反应参数, 如凝胶的组 成、模板剂的几何和电性质参数、溶剂的参数等等. 进一步采用决策树、支持相量机等数据分析方法，建 立特定的结构特征与合成反应参数之间的关系. 为实 现微孔晶体材料的定向设计合成开辟一条新的途径.

致谢本工作得到国家自然科学基金(批准号: 20871051)、国家重大基础研究发展规划项目(批准号: 2006CB806103)和国家重大国际合作研究项目(批准号: 20720102039)资助，特此一并致谢.

\section{参考文献}

1 Wilson S T, Lok B M, Messina C A, Cannan T R, Flanigen E M. Aluminophosphate molecular sieves: A new class of microporous crystalline inorganic solids. J Am Chem Soc, 1982, 104(4): 1146 - 1147 [DOI]

2 Bennett J M, Dyttych W J, Pluth J J. Structural features of aluminophosphate materials with Al/P = 1. Zeolites, 1986, 6(9): 349-360[DO]

$3 \mathrm{Yu} \mathrm{J} \mathrm{H,} \mathrm{Xu} \mathrm{R} \mathrm{R.} \mathrm{Insight} \mathrm{into} \mathrm{the} \mathrm{construction} \mathrm{of} \mathrm{open-framework} \mathrm{aluminophosphates.} \mathrm{Chem} \mathrm{Soc} \mathrm{Rev,} \mathrm{2006,} \mathrm{35(7):} \mathrm{593-604}$ 
4 Thomas J M, Raja R, Sankar G, Bell R G. Molecular-sieve catalysts for theselective oxidation of linear alkanes by molecular oxygen. Nature, 1999, 398, 227-230 [DOI]

5 Davis M E, Saldarriaga C, Montes C, Garces J, Crowder C. A molecular sieve with eighteen-membered rings. Nature, 1988, 331: 698-699[DOD]

6 Song X W, Li Y, Gan L, Wang Z P, Yu J H, Xu R R. Heteroatom-stabilized chiral framework of aluminophosphate molecular sieves. Angew Chem Int Ed, 2009, 48(2): 314-317 $\underline{\text { DOI] }}$

$7 \mathrm{Yu} \mathrm{J} \mathrm{H,} \mathrm{Xu} \mathrm{R} \mathrm{R.} \mathrm{Rich} \mathrm{structure} \mathrm{chemistry} \mathrm{in} \mathrm{the} \mathrm{aluminophosphate} \mathrm{family.} \mathrm{Acc} \mathrm{Chem} \mathrm{Res,} \mathrm{2003,} \mathrm{36(7):} \mathrm{481-490}$

8 Xing H Z, Li J Y, Yan W F, Chen P, Jin Z, Yu J H, Dai S, Xu R R. Cotemplating ionothermal synthesis of a new open-framework aluminophosphate with unique Al/P ratio of 6/7. Chem Mater, 2008, 20: 4179-4181 [DOI]

9 Huo Q S, Xu R R, Li S G, Ma Z G, Thomas J M, Jones R H, Chippindale A M. Synthesis and characterization of a novel extra large ring of aluminophosphate JDF-20. J Chem Soc Chem Commun, 1992, 875-876

10 Yan W F, Yu J H, Xu R R, Zhu G S, Xiao F S, Han Y, Kazumasa S, Osamu T. $\left[\mathrm{Al}_{12} \mathrm{P}_{13} \mathrm{O}_{52}\right]^{3-}\left[\left(\mathrm{CH}_{2}\right)_{6} \mathrm{~N}_{4} \mathrm{H}_{3}\right]^{3+}:$ An anionic aluminophosphate molecular sieve with Brönsted acidity. Chem Mater, 2000, 12(9): 2517-2519 [DOI]

$11 \mathrm{Yu} \mathrm{J} \mathrm{H,} \mathrm{Xu} \mathrm{R} \mathrm{R.} \mathrm{Toward} \mathrm{the} \mathrm{rational} \mathrm{design} \mathrm{and} \mathrm{synthesis} \mathrm{of} \mathrm{inorganic} \mathrm{microporous} \mathrm{and} \mathrm{related} \mathrm{materials.} \mathrm{Stud} \mathrm{Surf} \mathrm{Sci} \mathrm{Catal,} \mathrm{2004,}$ 154: $1-13$ [DOI]

12 Corma A. Towards a rationalization of zeolite and zeolitic materials synthesis. Stud Surf Sci Catal, 2004, 154: 25-40[DOI]

13 Li Y, Yu J H, Liu D H, Yan W F, Xu R R, Xu Y. Design of zeolite frameworks with defined pore geometry through constrained assembly of atoms. Chem Mater, 2003, 15: 2780-2785[DOI]

14 Li Y, Yu J H, Wang Z P, Zhang J N, Guo M, Xu R R. Design of chiral zeolite frameworks with specified channels through constrained assembly of atoms. Chem Mater, 2005, 17: 4399-4405[DOI]

15 Li Y, Yu J H, Xu R R, Baerlocher C, McCusker L B. Combining structure modeling and electron microscopy to determine complex zeolite framework structures. Angew Chem Int Ed, 2008, 47: 4401-4405[DOI]

16 Li Y, Yu J H, Jiang J X, Wang Z P, Zhang J N, Xu R R. Prediction of open-framework aluminophosphate structures using the automated assembly of secondary building units method with Lowenstein's constraints. Chem Mater, 2005, 17: 6086 - 6093[DOI]

17 Ren X Y, Li Y, Pan Q H, Yu J H, Xu R R, Xu Y. A crystalline germanate with mesoporous 30-ring channels. J Am Chem Soc, 2009, 131(40): 14128-14129[DOD]

18 Li J Y, Li L, Liang J, Chen P, Yu J H, Xu Y, Xu R R. Template-designed syntheses of open-framework zinc phosphites with extra-large 24-ring channels. Cryst Growth Des, 2008, 8(7): 2318-2323

19 Li J Y, Yu J H, Yan W F, Xu Y H, Xu W G, Qiu S L, Xu R R. Structures and templating effect in the formation of 2D layered aluminophosphates with $\mathrm{Al}_{3} \mathrm{P}_{4} \mathrm{O}_{16}^{3-}$ stoichiometry. Chem Mater, 1999, 11: 2600-2606 [DOD]

20 Yu J H, Li J Y, Wang K X, Xu R R, Sugiyama K, Terasaki O. Rational synthesis of microporous aluminophosphates with an inorganic open framework analogous to $\mathrm{Al}_{4} \mathrm{P}_{5} \mathrm{O}_{20} \mathrm{H} \cdot \mathrm{C}_{6} \mathrm{H}_{18} \mathrm{~N}_{2}$. Chem Mater, 2000, 12: 3783-3787 [DOI]

21 Li J Y, Yu J H, Xu R R. Database of AlPO syntheses. http://zeobank.jlu.edu.cn

22 Baerlocher Ch, McCusker L B. Database of zeolite structures. http://www.iza-structures.org/database/

\title{
Database of open-framework aluminophosphate syntheses: Introduction and application (I)
}

\author{
YAN Yan ${ }^{1}$, LI JiYang ${ }^{1}$, QI Miao ${ }^{2}$, ZHANG Xiao $^{1}$, YU JiHong ${ }^{1 *} \&$ XU RuRen ${ }^{* *}$ \\ 1 State Key Laboratory of Inorganic Synthesis and Preparative Chemistry, Jilin University, Changchun 130012, China; \\ 2 College of Chemistry, Northeast Normal University, Changchun 130117, China
}

Abstract: The database of open-framework aluminophosphate (AlPO) syntheses has been established, which includes about 1600 synthetic records. Data analysis has been done on the based of the framework composition, structrue dimension, pore ring, and organic template. This database will serve as useful guidance for the rational synthesis of microporous functional materials.

Keywords: aluminophosphates, open framework, synthesis, database 